\title{
MODELING THE DILATANCY OF OVERCONSOLIDATED CLAY
}

\author{
Zhiwei Gao* and Jidong Zhao \\ Department of Civil and Environmental Engineering, Hong Kong University of Science and \\ Technology, Clearwater Bay, Kowloon, Hong Kong \\ *email: gzwce@ust.hk
}

\begin{abstract}
Summary. The dilatancy of clay has long been considered as a function of the current stress state independent of the loading history. Experimental evidence, however, indicates the dilatancy behavior of over-consolidated (OC) clay bears close correlation with the overconsolidation ratio (OCR) of the soil. This paper presents a simple dilatancy relation for OC clays based on the modified Cam-clay (MCC) model. The dilatancy relation is integrated into a bounding surface model to predict the behavior of OC clays. With only three extra parameters added to the MCC model which can be easily calibrated by triaxial compression tests, the new model is shown to offer good predictions for the experimental data.
\end{abstract}

Keywords: Overconsolidation, clay, dilatancy, bounding surface model.

\section{INTRODUCTION}

The term dilatancy was first used by Reynolds [1] to describe the different behavior of volumetric change observed in dense and loose granular assemblies subjected to shear. Dilatancy has been regarded key to the characterization of strength and deformation of soils. Based on drained shear tests on sand, Taylor [2] has shown that there are two sources attributable to the peak strength of sand, one being the inter-particle friction and the other the dilatancy (or interlocking). Later, Rowe [3] developed his seminal stress-dilatancy theory highlighting the important contribution of dilatancy to soil strength and the mobilized friction in the loading process. Not uniquely tagged to sand only, stress dilatancy has been discussed for clay as well (e.g., [4]). In this study, a simple dilatancy relation for OC clay will be proposed based on the MCC model [5]. The new relation can be easily used in conjunction with either the bounding surface or the subloading surface models for OC clay. A demonstrative bounding surface model is developed with the implementation of this dilatancy relation.

\section{A SIMPLE DILATANCY RELATION FOR OC CLAY}

In several studies on OC clay using bounding surface, the ratio $R=r / \bar{r}$ has been used as a key variable to characterize the degree of overconsolidation, where $r$ and $\bar{r}$ denote the distances of the current and image stress point from the mapping center, respectively. Smaller $R$ corresponds to higher degree of overconsolidation. By adopting the origin of the $p-q$ plane as the mapping center, this $R$ will be employed as a descriptor for the degree of overconsolidation, 
where $p$ and $q$ denote the mean and deviatoric stress, respectively. Based on this definition of $R$, we propose the following dilatancy relation for OC clay

$$
D=\frac{d \varepsilon_{v}^{p}}{\left|d \varepsilon_{q}^{p}\right|}=\frac{M_{d}^{2}-\eta^{2}}{2 \eta}
$$

where

$$
M_{d}=M_{c} R^{m}
$$

where $\eta(=q / p)$ is the stress ratio; $M_{c}$ is the critical state stress ratio in triaxial compression and $m$ is a non-negative material constant. The dilatancy relation expressed above has the following features: (a) For Normally consolidated (NC) clays with the stress state on the bounding surface, Equation (2) is recovered to the dilatancy relation of the MCC model as $M_{d}=M_{c}$; (b) As the degree of overconsolidation increases, $R$ decreases, so do $M_{d}$ and $D$. This indicates that the response of a clay with higher degree of overconsolidation would be more dilative at the same stress ratio $\eta$ [6]; (c) At the critical state, the clay becomes $\mathrm{NC}$ and the stress state lies on the bounding surface ( $R=1$ ) with $\eta=M_{c}$, and thus, $D=0$.

\section{A BOUNDING SURFACE MODEL FOR OVERCONSOLIDATED CLAY}

Based on the dilatancy equation in Equations (1) and (2), a bounding surface model for OC clay is developed here. We will use the $\alpha-\gamma$ yield surface proposed by Collins [7] as the bounding surface with the assumption of $\alpha=\gamma$,

$$
\bar{f}=\frac{\left(\bar{p}-\alpha \bar{p}_{0} / 2\right)^{2}}{\left[(1-\alpha) \bar{p}+\alpha \bar{p}_{0} / 2\right]^{2}}+\frac{\bar{q}^{2}}{M_{c}^{2}\left[(1-\alpha) \bar{p}+\alpha^{2} \bar{p}_{0} / 2\right]^{2}}-1=0
$$

where $\bar{p}_{0}$ denotes the size of the bounding surface; $\bar{p}$ and $\bar{q}$ are respectively the mean stress and deviatoric stress at the image point. Following Dafalias and Herrmann [8], the same isotropic hardening law as in the MCC model is assumed for $\bar{p}_{0}$,

$$
d \bar{p}_{0}=\frac{1+e}{\lambda-\kappa} \bar{p}_{0} d \varepsilon_{v}^{p}
$$

where $\lambda$ and $\kappa$ are the compression and swelling indices, respectively; $d \varepsilon_{v}^{p}$ is the plastic volumetric strain increment. An associated flow rule in the deviatoric plane will be employed, 


$$
d \varepsilon_{q}^{p}=\langle d L\rangle \frac{\partial \bar{f}}{\partial \bar{q}}
$$

where $d L$ is the loading index; $d \varepsilon_{q}^{p}$ is the plastic deviatoric strain increment; \langle\rangle are the McCauley brackets. The plastic modulus at the image stress point, $\bar{K}_{p}$ is expressed as

$$
\bar{K}_{p}=-\frac{\partial f}{\partial \bar{p}_{0}} \frac{(1+e) \bar{p}_{0}}{(\lambda-\kappa)} D_{R=1}=-\frac{(1+e) \bar{p}_{0}}{(\lambda-\kappa)} \frac{\partial f}{\partial \bar{p}_{0}} \frac{\partial f}{\partial \bar{q}} \frac{\left(M_{c}^{2}-\bar{\eta}^{2}\right)}{2 \bar{\eta}}
$$

The expression for the plastic modulus at the current stress state $K_{p}$ is simply assumed to be of the identical form of $\bar{K}_{p}$ by simply replacing $M_{c}$ by a virtue peak stress ratio $M_{v}$,

$$
K_{p}=-\frac{(1+e) \bar{p}_{0}}{(\lambda-\kappa)} \frac{\partial f}{\partial \bar{p}_{0}} \frac{\partial f}{\partial \bar{q}} \frac{\left(M_{v}^{2}-\eta^{2}\right)}{2 \eta}
$$

where $M_{v}$ is related to $R$ according to

$$
M_{v}=M_{c} R^{-n}
$$

where $n$ is a non-negative model parameter. For OC clay, $K_{p}$ is dependent on the difference between the current stress ratio $\eta(=\bar{\eta})$ from the virtue peak stress ratio $M_{v}$ attainable at the current degree of overconsolidation defined by $R$. Since $R \leq 1$, the virtue peak stress ratio $M_{v}$ in Equation (11) is a decreasing function of $R$ and thus an increasing function with the degree of overconsolidation [6].

\section{MODEL VERIFICATION}

Shown in Fig. 1 is the comparison between our model predictions with the test data on the Boston blue clay (data from [6]). Good agreement can be observed. In the figures, $\varepsilon_{a}$ is the axial strain and $p_{c}$ is the maximum consolidation pressure. The model parameters are listed in Table 1 , where $v$ is the Poisson ratio and $\Gamma$ is the void ratio at the reference mean stress $p_{r}$ on the normal consolidation line.

\section{CONCLUSION}

A simple dilatancy relation for OC clay is proposed and used in a predictive bounding surface 
model. The model has been demonstrated to predict the behavior of OC clays well as compared to test data. Future work will be done on modeling the behavior of structured soils (e.g., [9]).

Table 1 Model parameters for Boston blue clay

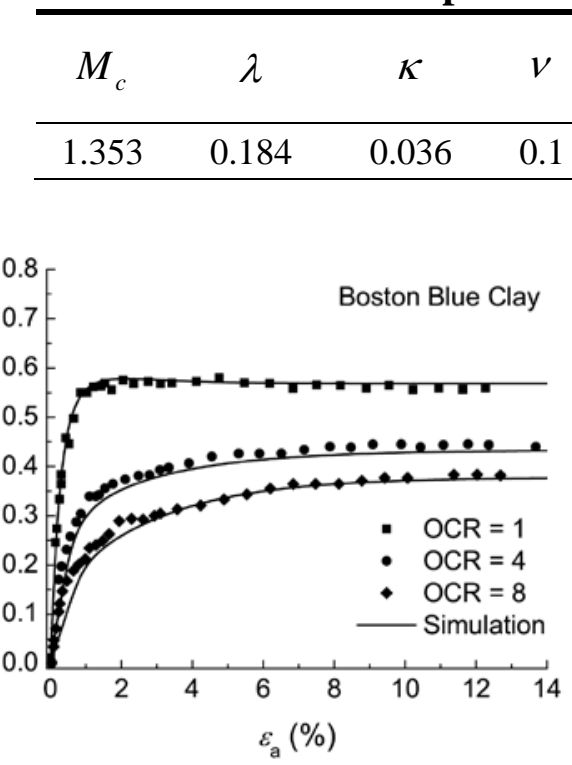

(a)

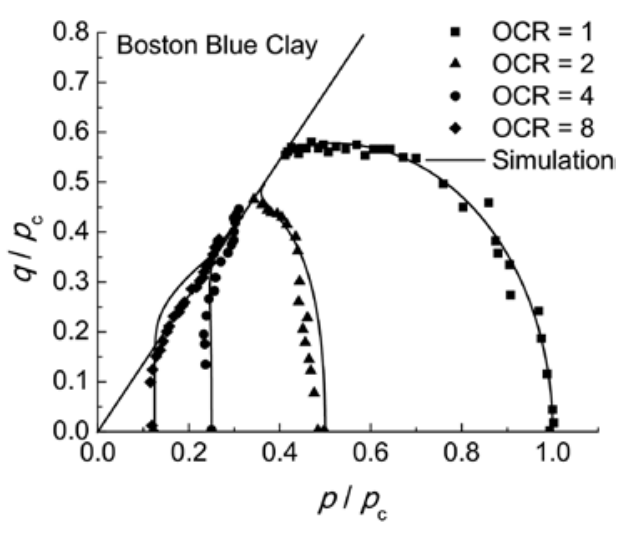

(b)

Fig. 1 Comparison between the model simulation and test results on Boston blue clay (data from Pestana et al. (2002)): (a) the $\varepsilon_{a}-q / p_{c}$ relations and (b) the effective stress paths

\section{ACKNOWLEDGEMENTS}

This work was supported by Research Grants Council of Hong Kong (under grant no. 623211).

\section{REFERENCES}

[1] Reynolds, O.: On the dilatancy of media composed of rigid particles in contact. With experimental illustrations. Phil. Mag. 20, 469-482 (1885)

[2] Taylor, D.W.: Fundamentals of soil mechanics. Wiley, New York. (1948)

[3] Rowe, P.W.: The stress-dilatancy relation for static equilibrium of an assembly of particles in contact. Proc. Roy. Soc. London. A269, 500-527 (1962)

[4] Schofield, A.N.: The Mohr-Coulomb error. Proc. Symp. On Mechanics and Geotechnics, LMS Ecole Polytechnique, Paris, 23, 19-27 (1998)

[5] Roscoe, K.H., Burland, J.B.: On the generalized stress-strain behavior of 'wet' clay. In: Engineering Plasticity, pp. 535-609. Cambridge University Press, Cambridge (1968)

[6] Pestana, J.M., Whittle, A.J., Gens, A.: Evaluation of a constitutive model for clays and sands: Part II - clay behaviour. Int. J. Numer. Anal. Meth.Geomech. 26, 1123-1146 (2002)

[7] Collins, I.F.: Elastic/plastic models for soils and sands. Int. J. Mech. Sci. 47, 493-508 (2005)

[8] Dafalias, Y.F., Herrmann, L.R.: Bounding surface plasticity II: Application to isotropic cohesive soils. J. Eng. Mech. 112(12), 1263-1291 (1986)

[9] Gao, Z.W., Zhao, J.D.: Constitutive modeling of artificially cemented sand by considering fabric anisotropy. Computer and Geotechnics 41, 57-69 (2012) 